\title{
DETERMINACIÓN DE LOS PARÁMETROS ÓPTIMOS PARA LA MENESTRA PRECOCIDA Y DESHIDRATADA A PARTIR DE FRIJOL CASTILLA (Vigna unguiculata)
}

DETERMINATION OF THE OPTIMAL PARAMETERS FOR PRECOOKED AND DEHYDRATED MENESTRA FROM CASTILLA BEANS (Vigna unguiculata)

Alfredo Lázaro Ludeña Gutiérrez ${ }^{1}$

Henry Joel Aquino Silva ${ }^{2}$

Wilfredo Trasmonte Pinday ${ }^{3}$ Percy Permín Velázquez $\mathrm{Ccosi}^{4}$

Información del artículo: Recibido: 25/04/2020 Aceptado: $11 / 10 / 2020$

1,2 Docente Ingeniería Agroindustrial e Industrias Alimentarias, Universidad Nacional de Piura 3,4

Docente Ingeniería en Industrial Alimentarias e Ingeniería Agroindustrial, Universidad Nacional San Cristóbal de Huamanga

E-mail: ${ }^{1}$ ludesall@hotmail.com, ${ }^{2 h}$ jaquinos@gmail.com, ${ }^{3}$ wilfredo.trasmonte@unsch.edu.pe, percy.velasquez@unsch.edu.pe 


\section{Resumen}

La investigación se desarrolló para determinar los parámetros óptimos para la obtención de una menestra precocida y deshidratada a partir de frijol castilla. Se realizó el análisis del flujo de proceso, se determinó el porcentaje de humedad, tiempo de rehidratación, evaluación de perfil microbiológico, físico-químico y el análisis sensorial. Se analizaron tiempos de precocción a 30, 40 y 50 min y tiempos de deshidratación a 3, 4 y $5 \mathrm{~h}$ a condiciones constantes de temperatura $\left(65^{\circ} \mathrm{C}\right)$ y velocidad de aire $(2 \mathrm{~m} / \mathrm{s})$. Se realizó el análisis estadístico de las muestras del proceso, determinándose el más adecuado, mediante un análisis de diseño bifactorial $3 \times 3$ con 3 repeticiones, que determinó el flujo de proceso para la obtención de la menestra precocida y deshidratada, considerando: $6 \mathrm{~h}$ de remojo, 40 min de precocción y $5 \mathrm{~h}$ de deshidratación. La muestra seleccionada reportó los siguientes resultados: $356,57 \mathrm{Kcal} / 100 \mathrm{~g}, 2,51 \%$ de ceniza, 22,14\% de proteína, 3,61 \% de grasa, 58,88 $\%$ de carbohidratos y $5,8 \%$ de fibra; el análisis sensorial se realizó mediante una prueba afectiva, midiéndose los siguientes atributos: olor, color, sabor, textura y aceptabilidad, con un panel de 10 jueces habituales; los resultados estadísticos indicaron que al finalizar la evaluación no existía diferencia significativa en cuanto a olor, color, sabor y textura, teniendo mejor aceptación el tratamiento 6 ( $40 \mathrm{~min}$ de cocción y $5 \mathrm{~h}$ de deshidratado) por conservar mejor sus características sensoriales comparado con los demás tratamientos.

Palabras claves: Frijol: Cocción; Deshidratación; Humedad; Rehidratación.

\section{Abstract}

The research was developed to determine the optimal parameters for obtaining a precooked and dehydrated of Castilla beans. The analysis of the process flow was carried out, the percentage of humidity, rehydration time, evaluation of the microbiological and physical-chemical profile and the sensory analysis were determined. Precooking times were analyzed at 30, 40 and $50 \mathrm{~min}$ and dehydration times at 3,4 and $5 \mathrm{~h}$ at constant temperature $\left(65^{\circ} \mathrm{C}\right)$ and air speed $(2 \mathrm{~m} / \mathrm{s})$. The statistical analysis of the process samples was carried out, determining the most suitable one, by means of a $3 \times 3$ bifactorial design analysis with 3 repetitions, which determined the process flow to obtain the precooked and dehydrated beans, considering: $6 \mathrm{~h}$ of soaking, 40 minutes of pre-cooking and $5 \mathrm{~h}$ of dehydration. The selected sample reported the following results: $356.57 \mathrm{Kcal} / 100 \mathrm{~g}, 2.51 \%$ ash, $22.14 \%$ protein, $3.61 \%$ fat, $58.88 \%$ carbohydrates and $5.8 \%$ fiber; the sensory analysis was done by an affective test, measuring the following attributes: odour, colour, taste, texture and acceptability, with a panel of 10 usual judges; the statistical results indicated that at the end of the evaluation there was no significant difference in odour, colour, taste and texture, with treatment 6 having better acceptance (40 min of cooking and $5 \mathrm{~h}$ of dehydration) because it conserves better its sensory characteristics compared to the other treatments.

Keywords: Beans, Cooking; Dehydration; Humidity; Rehydration 


\section{Introducción}

El frijol castilla (Vigna unguiculata), es un producto considerado de alta calidad en el mundo, se exporta a más de 40 países, aspectos que aseguran su crecimiento y potencial exportador sobre todo a España, Estados Unidos, Japón, Grecia, Italia, Holanda, Puerto Rico y Portugal. La ventaja comparativa que posee este producto se debe a que es de consumo masivo y que forma parte de la canasta básica familiar de los niveles socioeconómicos medios y bajos, por lo que representa en los mercados internacionales una alta tendencia de la demanda. Para la salud trae beneficios resaltantes tales como: fácil digestión, eliminación de agua y toxinas del cuerpo, contrarresta la diabetes, según Chacon (2010). El crecimiento poblacional y el suministro de alimentos crean la necesidad de enfocar investigaciones hacia la identificación y evaluación de nuevas fuentes nutricionales. En vista de lo anterior, las leguminosas se perfilan como buenos recursos proteicos y de otros nutrientes de fácil acceso y bajo precio, siendo una alternativa para mitigar la mal nutrición presentada en los países de tercer mundo (Vargas, 2012). La producción agrícola del frijol castilla en la región Piura - Perú, ha representado la principal actividad para el desarrollo social económico de la población rural; siendo este un producto exportable ya que cuenta con una amplia demanda internacional, además de poder ser procesado, enlatado y congelado..

\section{Objetivos}

Determinar los parámetros óptimos para la obtención de una menestra precocida y deshidratada a partir de frijol castilla (Vigna unguiculata).

\section{Metodología}

Se sometió el frijol castilla a diferentes tiempos de precocción (30, 40 y 50 minutos) y deshidratación ( 3,4 y 5 horas) a temperatura constante de $65^{\circ} \mathrm{C}$, para la obtención de una menestra precocida y deshidratada. Se experimentó a través de muestreos, que menestra precocida y deshidratada a partir del frijol castilla se logra los parámetros óptimos en cuanto a sus características fisicoquímicas, microbiológicas y sensoriales en el producto final. Se aplicó un diseño experimental bifactorial $3 \times 3$, con un nivel de significancia de 0,05 con tres repeticiones y se determinó en todos los tratamientos el porcentaje de humedad y el tiempo de rehidratación. Todas las operaciones se realizaron con buenas prácticas de manufactura teniendo en cuenta la higiene y sanidad de la materia prima y del proceso de elaboración.

El frijol castilla utilizado para el estudio se adquirió a granel de los almacenes de acopio del Distrito de La Unión -Piura - Perú, de la cosecha de 2017, con las características de madurez homogénea (color, dureza y tamaño) y libre de defectos. Se estableció las condiciones de la materia prima utilizada de acuerdo a lo estipulado en la NTP 205.015 (2015) para el frijol castilla, Norma Técnica Peruana que establece los requisitos que debe cumplir una leguminosa seca para poder ser destinada a consumo humano. 


\section{Resultados}

La figura 1 muestra el incremento de peso de frijol castilla en función del tiempo de secado, donde se puede observar la ganancia de peso a temperatura ambiente. El remojo es una fase preliminar común a casi todos los métodos de preparación de las leguminosas.

\section{Figura 1}

Incremento de peso en la rehidratación de frijol castilla en función al tiempo de remojo

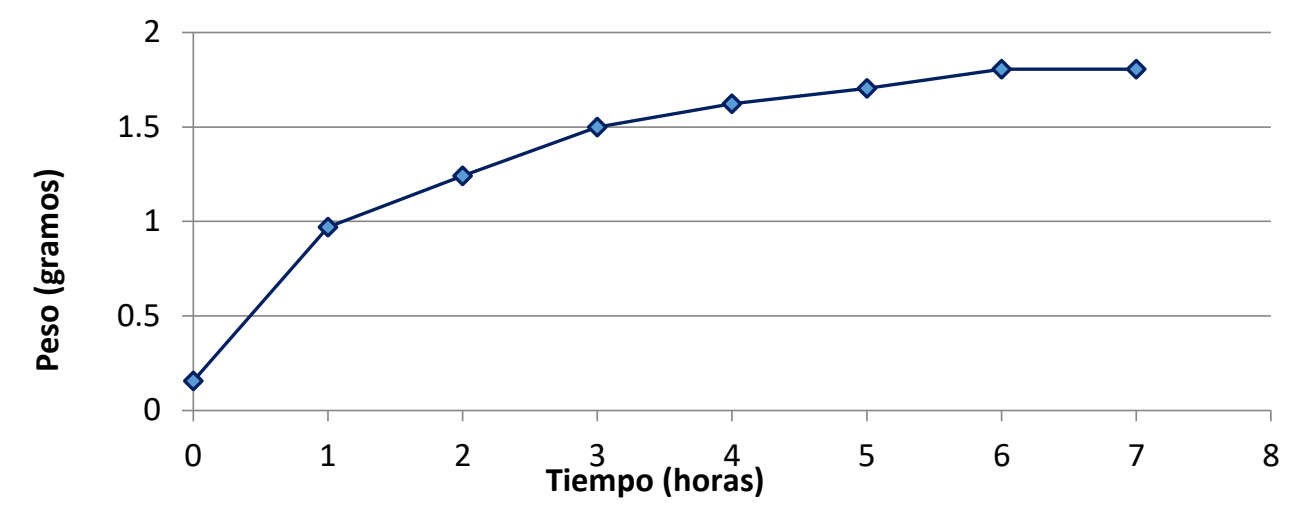

Nota. Elaboración propia.

En la figura 2, se observa el comportamiento típico de la variación de humedad total en $\mathrm{gH} 2 \mathrm{O} / \mathrm{g}$ sólidos secos, frente al tiempo transcurrido en minutos; en ella se aprecian los tres períodos de secado antes mencionados correspondiendo el tramo recto al período constante (Geankoplis, 1998). Esta curva es asintótica con el eje horizontal. Se muestra en la figura 2 , el tiempo óptimo de secado es de 300 minutos, observándose que el porcentaje de humedad en base seca de la menestra precocida y deshidratada es de 0,1540 gH2O/g sólidos equivalente a 0,1286 en base húmeda, valor establecido dentro de la NTP 205.015 (2015) para el frijol castilla.

Figura 2

Curva de secado en función del contenido de humedad y el tiempo

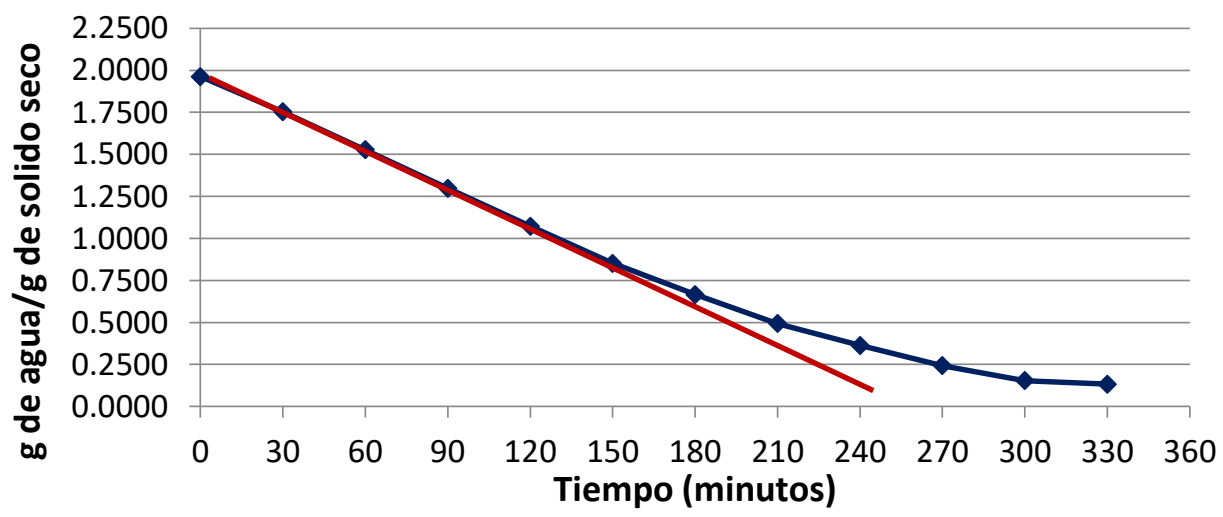

Nota. Elaboración propia. 


\section{Tabla 1}

Resultados obtenidos del frijol precocido y deshidratado

\begin{tabular}{ll}
\hline \multicolumn{1}{c}{ Parámetros } & \multicolumn{1}{c}{ Resultados } \\
\hline$W_{\text {total frijol castilla (materia }}$ & $1,000 \mathrm{~kg}$ \\
$\mathrm{~W}_{\text {total frijol precocido }}$ & $2,500 \mathrm{~kg}$ \\
$\mathrm{~W}_{\text {de agua en el frijol }}$ & $1,656 \mathrm{~kg}$ de agua \\
$\mathrm{W}_{\text {sólido seco }}$ & $0,844 \mathrm{~kg}$ \\
$\mathrm{X}_{0}$ & $\mathrm{X}_{0}=1,9621 \frac{\mathrm{kg} \text { de agua }}{\mathrm{kg} \text { de solido seco }}$ \\
$\mathrm{X}_{1}$ & $\mathrm{X}_{1}=0,154 \frac{\mathrm{kg} \text { de agua }}{\mathrm{kg} \text { de solido seco }}$ \\
$\mathrm{W}_{\text {agua evaporada }}$ & $1,526 \mathrm{~kg}$ de agua evaporada. \\
$\mathrm{W}_{\text {agua ligada }}$ & $0,13 \mathrm{~kg}$ de agua ligada \\
W $_{\text {frijol deshidratado }}$ & $0,974 \mathrm{~kg}$ de frijol deshidratado \\
Tiempo & 5 horas \\
\hline
\end{tabular}

Nota. Elaboración propia.

De los datos obtenidos se concluye que por $2,500 \mathrm{~kg}$ de frijol precocido se obtiene 0,974 kg de frijol deshidratado y se necesitan 5 horas para obtener la deshidratación de esa cantidad de frijol castilla.

El frijol castilla empieza con una humedad de 1,9621 $\frac{\mathrm{kg} \text { de agua }}{\mathrm{kg} \text { de sólido seco }}$ y terminando el proceso de deshidratado final es $0,154 \frac{\mathrm{kgde} \text { agua }}{\mathrm{kgde \text {sólidoseco }}}$.

La humedad crítica, se puede observar en la figura 2, ocurre a los $150 \mathrm{~min}$, por lo cual desde que inicia el proceso de secado hasta llegar a los $150 \mathrm{~min}$ la velocidad de secado es constante. Finalmente se aprecia en una segunda etapa (periodo de velocidad decreciente) más lentas para valores de humedad del producto menores a humedad crítica, esta etapa tiene una duración de 270 minutos.

En la figura 3, se presenta la variación de la velocidad de secado " $\mathrm{R}$ " expresada en $\mathrm{gH}_{2} \mathrm{O}$ / $\mathrm{m}^{2}$.min, frente a la humedad libre promedio visualizándose los tres períodos de secado durante el proceso de secado de deshidratado de la menestra precocida y deshidratada (Fang, 2007), para el proceso de secado de arroz. 


\section{Figura 3}

Variación de la velocidad de secado " $R$ " en función de la humedad

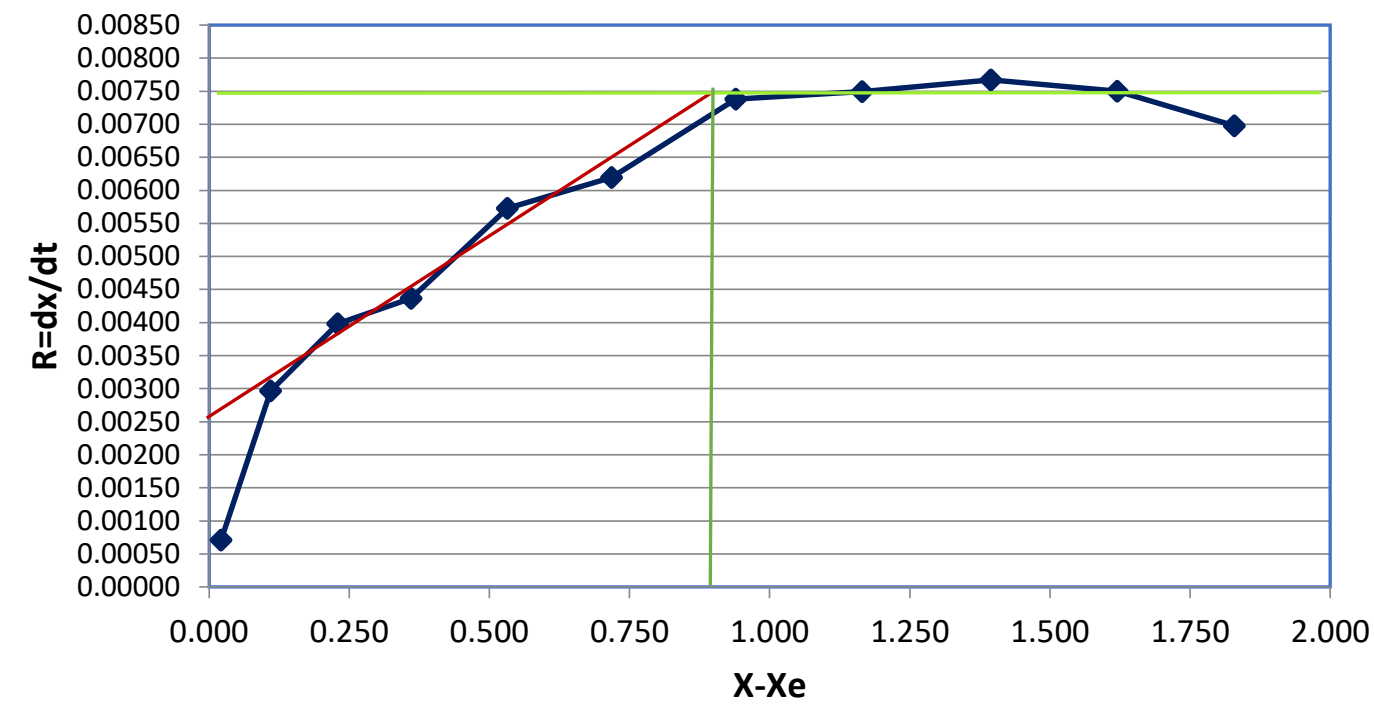

Nota. Elaboración propia.

En la tabla 2 se muestra los resultados de la influencia del tiempo de precocción y el tiempo de deshidratación en el porcentaje de humedad de la menestra precocida y deshidratada a partir de fríjol castilla.

Tabla 2

Diseño de bloques

\begin{tabular}{clll}
\hline \multirow{2}{*}{$\begin{array}{l}\text { Tiempo } \\
\text { pre-cocción }\end{array}$} & \multicolumn{3}{c}{ Tiempo de deshidratación } \\
\cline { 2 - 4 } 30 & $\mathbf{3}$ & $\mathbf{4 h}$ & $\mathbf{5 h}$ \\
\hline \multirow{3}{*}{$30 \mathrm{~min}$} & 43,52 & 29,51 & 14,51 \\
& 44,15 & 28,75 & 13,02 \\
& 42,55 & 29,12 & 14,89 \\
\hline \multirow{3}{*}{40 min } & 42,01 & 26,54 & 10,95 \\
& 40,12 & 23,03 & 11,04 \\
& 41,14 & 25,98 & 12,51 \\
\hline \multirow{3}{*}{50 min } & 43,35 & 24,17 & 10,17 \\
& 26,04 & 26,04 & 9,74 \\
& 25,53 & 25,53 & 10,23 \\
\hline
\end{tabular}

Nota. Elaboración propia.

La tabla 3 muestra el análisis de varianza que permitió comparar el porcentaje de humedad, utilizando diferentes tiempos de precocción, tiempos de deshidratación y tratamientos; la prueba permitió contrastar la hipótesis de que el porcentaje de humedad no difiere significativamente al utilizar los tres tiempos de pre-cocción, tres tiempos de deshidratación o la combinación de ambos tratamientos. 
Tabla 3

Análisis de varianza del porcentaje de humedad en función del tiempo de precocción y tiempo de deshidratación

\begin{tabular}{|c|c|c|c|c|c|}
\hline F.V. & $\begin{array}{l}\text { Suma de } \\
\text { cuadrados }\end{array}$ & G.L. & $\begin{array}{l}\text { Media } \\
\text { cuadrática }\end{array}$ & $\mathbf{F}$ & Sig. \\
\hline bloque & 3,942 & 2 & 1,971 & 2,289 & 134 \\
\hline $\begin{array}{l}\text { Tiempo de pre } \\
\text { cocción(A) }\end{array}$ & 52,492 & 2 & 26,246 & 30,474 & ,000 \\
\hline $\begin{array}{l}\text { Tiempo de } \\
\text { Deshidratación(B) }\end{array}$ & 4173,966 & 2 & 2086,983 & 2423,192 & ,000 \\
\hline Factor $A *$ factor $B$ & 12,166 & 4 & 3,042 & 3,532 & ,030 \\
\hline Error & 13,780 & 16 & ,861 & & \\
\hline Total corregida & 4256,347 & 26 & & & \\
\hline
\end{tabular}

Nota. Elaboración propia.

La tabla 4 muestra la prueba de Duncan al 5\% del porcentaje de humedad en función del tiempo de precocción.

Tabla 4

Prueba de Duncan del porcentaje de humedad en función del tiempo de precocción

\begin{tabular}{llcc}
\hline $\begin{array}{l}\text { Tiempo de } \\
\text { precocción (A) }\end{array}$ & Promedio & $\mathbf{1}$ & Subconjunto \\
\cline { 3 - 4 } & & $\mathrm{2}$ & \\
\hline $40 \mathrm{~min}$ & 25,9244 & $\mathrm{a}$ & \\
$50 \mathrm{~min}$ & 25,9422 & & $\mathrm{~b}$ \\
$30 \mathrm{~min}$ & 28,8911 & & \\
\hline
\end{tabular}

Nota. Elaboración propia.

La tabla 5 muestra la prueba de Duncan al 5\% del porcentaje de humedad en función del tiempo de deshidratación.

\section{Tabla 5}

Prueba de Duncan al 5\% del porcentaje de humedad en función del tiempo de deshidratación

\begin{tabular}{llccc}
\hline $\begin{array}{c}\text { Tiempo de } \\
\text { Deshidratación } \\
\text { (B) }\end{array}$ & Promedios & \multicolumn{3}{c}{ Subconjunto } \\
\cline { 3 - 5 } & & a & $\mathbf{2}$ & $\mathbf{3}$ \\
\hline $\mathrm{h}$ & 11,8956 & & $\mathrm{~b}$ & \\
$\mathrm{~h}$ & 26,5189 & & & $\mathrm{c}$ \\
$3 \mathrm{~h}$ & 42,3433 & & & \\
\hline
\end{tabular}

Nota. Elaboración propia. 
La tabla 6 muestra la prueba de Duncan al 5\% del porcentaje de humedad para los efectos de interacción de tiempo de pre-cocción y tiempo de deshidratación.

\section{Tabla 6}

Prueba de Duncan al 5\% del porcentaje de humedad para efectos de interacción de tiempo de pre-cocción y tiempo de deshidratación

\begin{tabular}{cccc}
\hline \multirow{2}{*}{$\begin{array}{c}\text { Tiempo pre- } \\
\text { cocción (min) }\end{array}$} & \multicolumn{3}{c}{$\begin{array}{c}\text { tiempo de } \\
\text { deshidratación (h) }\end{array}$} \\
\cline { 2 - 4 } & $\mathbf{3}$ & $\mathbf{4}$ & $\mathbf{5}$ \\
\hline $\mathbf{3 0}$ & $43,41(\mathrm{f})$ & $29,13(\mathrm{~d})$ & $14,14(\mathrm{~b})$ \\
$\mathbf{4 0}$ & $41,09(\mathrm{e})$ & $25,18(\mathrm{c})$ & $11,50(\mathrm{a})$ \\
$\mathbf{5 0}$ & $42,53(\mathrm{ef})$ & $25,25(\mathrm{c})$ & $10,05(\mathrm{a})$
\end{tabular}

Nota. Elaboración propia.

En la figura 4 se muestra el promedio del porcentaje de humedad en función del tiempo de precocción y tiempo de deshidratación.

\section{Figura 4}

Promedio del porcentaje de humedad en función del tiempo de precocción y deshidratación

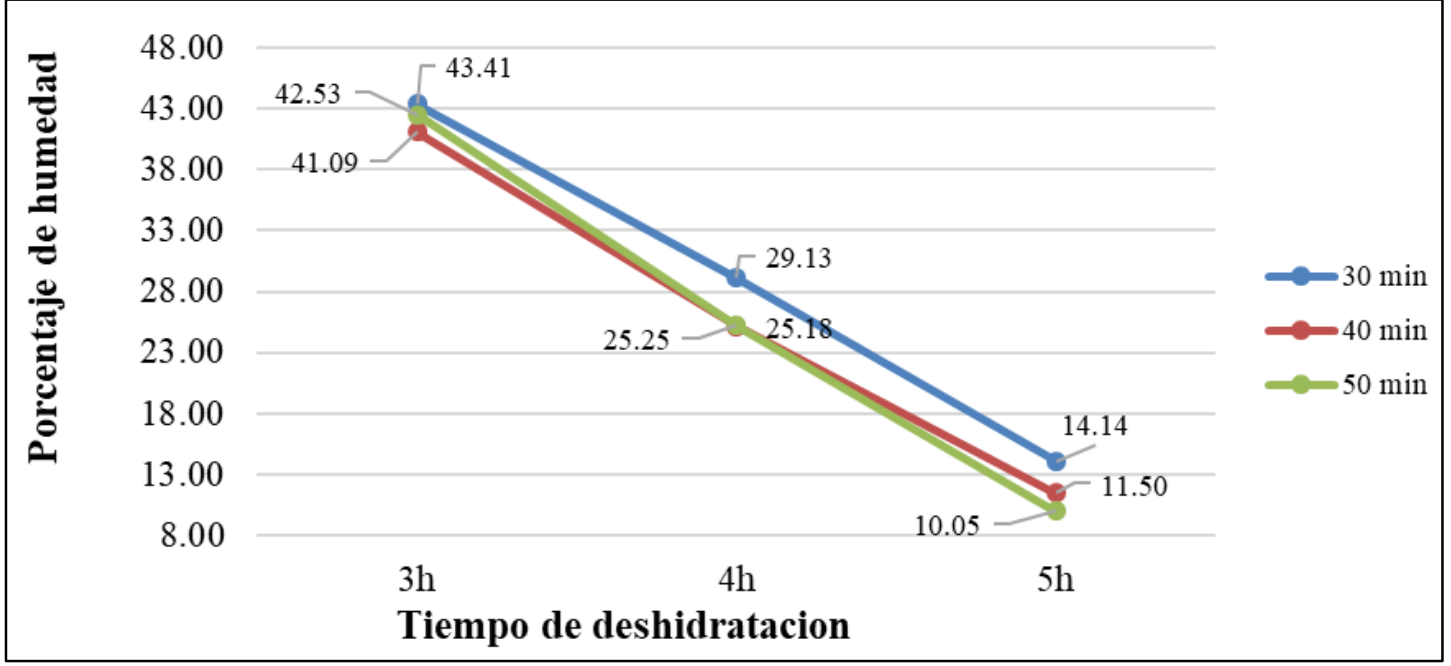

Nota. Elaboración propia.

En la tabla 7 se muestra el diseño de bloques de la influencia del tiempo de precocción y tiempo de deshidratación en el tiempo de rehidratación de la menestra precocida y deshidratada a partir de frijol castilla. 
Tabla 7

Diseño de bloques para el tiempo de rehidratación

\begin{tabular}{clcc}
\cline { 2 - 4 } \begin{tabular}{c} 
Tiempo \\
pre-cocción \\
\cline { 2 - 4 }$(\mathbf{m i n})$
\end{tabular} & $\mathbf{3}$ & $\mathbf{4}$ & $\mathbf{5}$ \\
\cline { 2 - 4 } 30 & 15,35 & 10,35 & 9,11 \\
& 16,51 & 12,02 & 8,35 \\
& 17,02 & 9,50 & 8,52 \\
\hline \multirow{2}{*}{40} & 6,02 & 6,25 & 6,30 \\
& 7,13 & 7,08 & 5,57 \\
& 6,35 & 5,57 & 6,15 \\
\hline \multirow{2}{*}{50} & 6,02 & 5,12 & 5,14 \\
& 5,03 & 6,29 & 6,18 \\
& 5,11 & 5,06 & 5,25 \\
\hline
\end{tabular}

Nota. Elaboración propia.

En la tabla 8 se muestra el ANVA que permitió comparar el tiempo de rehidratación, utilizando diferentes tiempos de precocción, tiempos de deshidratación y tratamientos.

Tabla 8

ANVA del tiempo de rehidratación en función del tiempo de precocción y tiempo de deshidratación

\begin{tabular}{|c|c|c|c|c|c|}
\hline Origen & $\begin{array}{l}\text { Suma de } \\
\text { cuadrados } \\
\text { tipo III }\end{array}$ & gl & $\begin{array}{l}\text { Media } \\
\text { cuadrática }\end{array}$ & $\mathbf{F}$ & Sig. \\
\hline Bloque & 1,971 & 2 & 0,986 & 2,128 & 0,151 \\
\hline $\begin{array}{l}\text { Tiempo de } \\
\text { precocción(A) }\end{array}$ & 218,256 & 2 & 109,128 & 235,651 & 0,000 \\
\hline $\begin{array}{l}\text { Tiempo de } \\
\text { Deshidratación(B) }\end{array}$ & 34,013 & 2 & 17,006 & 36,723 & 0,000 \\
\hline Factor $(A) *$ factor $(B)$ & 60,659 & 4 & 15,165 & 32,747 & 0,000 \\
\hline Error & 7,409 & 16 & 0,463 & & \\
\hline Total corregida & 322,308 & 26 & & & \\
\hline
\end{tabular}

Nota. Elaboración propia.

En la tabla 9 se muestra la Prueba de Duncan al 5\% de tiempo de rehidratación en función del tiempo de precocción. 
Tabla 9

Prueba de Duncan al 5\% de tiempo de rehidratación en función del tiempo de precocción

\begin{tabular}{lllcc}
\hline \multirow{2}{*}{$\begin{array}{c}\text { Tiempo de Pre cocción } \\
\text { (min) }\end{array}$} & Promedios & $\mathbf{1}$ & $\mathbf{2}$ & $\mathbf{3}$ \\
\cline { 3 - 5 } & 5,4667 & $\mathrm{a}$ & & \\
\hline 50 & 6,2689 & & $\mathrm{~b}$ & \\
30 & 11,8589 & & & $\mathrm{c}$ \\
\hline
\end{tabular}

Nota. Elaboración propia.

En la tabla 10 se muestra la prueba de Duncan al 5\% del tiempo de rehidratación en función del tiempo de deshidratación.

Tabla 10

Prueba de Duncan al 5\% del tiempo de rehidratación en función del tiempo de deshidratación

\begin{tabular}{cllll}
\hline $\begin{array}{c}\text { Tiempo de Deshidratación } \\
\text { (h) }\end{array}$ & Promedios & $\begin{array}{l}\text { Subconjunto } \\
\mathbf{1}\end{array}$ & $\mathbf{2}$ & $\mathbf{3}$ \\
\hline $5 \mathrm{~h}$ & 6,7300 & $\mathrm{a}$ & & \\
$4 \mathrm{~h}$ & 7,4711 & & $\mathrm{~b}$ & \\
$3 \mathrm{~h}$ & 9,3933 & & & $\mathrm{c}$ \\
\hline
\end{tabular}

Nota. Elaboración propia.

En la tabla 11 se muestra la prueba de Duncan al 5\% del tiempo de rehidratación para efectos de interacción de tiempo de precocción y tiempo de deshidratación.

Tabla 11

Prueba de Duncan al $5 \%$ del tiempo de rehidratación para efectos de interacción de tiempo de precocción y tiempo de deshidratación

\begin{tabular}{lrcc}
\hline \multirow{2}{*}{$\begin{array}{c}\text { Tiempo de pre } \\
\text { cocción (min) }\end{array}$} & $\mathbf{3}$ & \multicolumn{3}{c}{ Tiempo de deshidratación (h) } \\
\cline { 2 - 4 } & 16,29 (d) & 10,62 (c) & 8,66 (b) \\
\hline 30 & 6,50 (a) & 6,30 (a) & 6,01 (a) \\
50 & 5,39 (a) & 5,49 (a) & 5,52 (a) \\
\hline
\end{tabular}

Nota. Elaboración propia.

La figura 5 muestra el promedio del tiempo de rehidratación en función del tiempo de precocción y deshidratación. 


\section{Figura 5}

Promedio de tiempo de rehidratación en función del tiempo de precocción y deshidratación

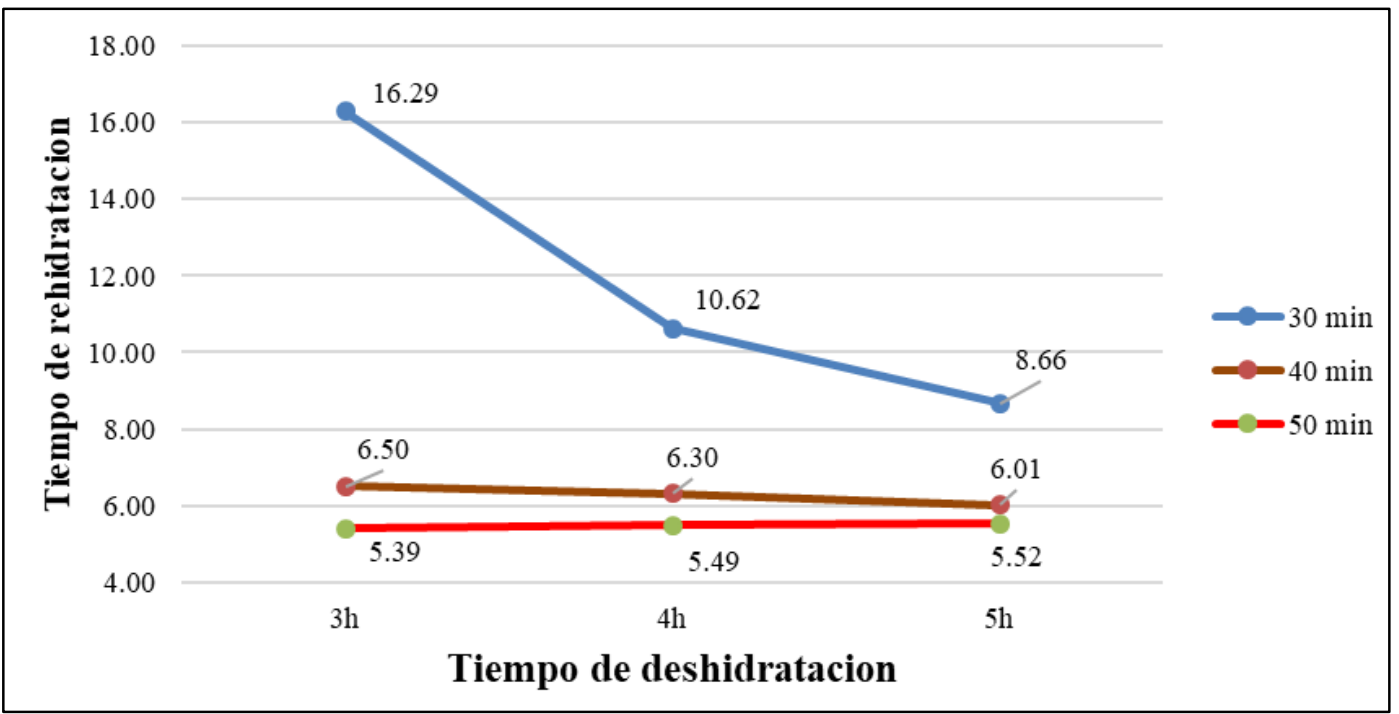

Nota. Elaboración propia.

En la tabla 12 se muestra los resultados del análisis químico del frijol castilla precocido y deshidratado.

\section{Tabla 12}

Valores del análisis químico de la muestra de menestra precocida y deshidratada

\begin{tabular}{ll}
\hline Parámetros de evaluación & Valor \\
\hline Energía total (Kcal/100g) & 356,57 \\
Humedad (\%) & 12,86 \\
Cenizas (\%) & 2,51 \\
Proteínas (\%) & 22,14 \\
Grasas total (\%) & 3,61 \\
Carbohidratos (\%) & 58,88 \\
Fibra (\%) & 5,8 \\
pH a 28 ${ }^{\circ} \mathrm{C}$ (unidad de pH) & 6,6 \\
Acidez(\%) Expresada como ácido sulfúrico & 0,18 \\
\hline
\end{tabular}

Nota. Informe técnico emitido por el laboratorio de control de calidad de la FIP - UNP.

En la tabla 13 se muestra los resultados de las pruebas microbiológicas después de la menestra precocida y deshidratada de frijol castilla, la muestra fue envasada en envase de polietileno de baja densidad y expuesta al medio ambiente. 


\section{Tabla 13}

Valores de crecimiento de microorganismos de la muestra final de menestra precocida y deshidratada

\begin{tabular}{lcc}
\hline \multicolumn{1}{c}{ Ensayos } & Resultados & Parámetro \\
\hline Aerobios mesofilos (ufc/g) & $12 \times 10$ & $10^{4}$ \\
Mohos (ufc/g) & 12 & $10^{3}$ \\
Levaduras(ufc/g) & 0 & $10^{3}$ \\
Coliformes totales (NMP/g) & $<3$ & $10^{2}$ \\
Salmonella sp (Ausencia/25g) & 0 & Ausencia \\
\hline
\end{tabular}

Nota. Informe técnico emitido por el laboratorio de control de calidad de la FIP - UNP.

Se realizó la evaluación sensorial de los nueve tratamientos con diferentes tiempos de precocción y deshidratado de la menestra precocida y rehidratada en los atributos de color, olor sabor, textura y aceptabilidad. En la figura 6 se muestran los efectos de interacción del tiempo de precocción vs tiempo de deshidratación para el color de la menestra precocida y deshidratada, resultado de la prueba de Duncan al $5 \%$.

\section{Figura 6}

Calidad de Color para efectos de interacción de Tiempo de Pre cocción * Tiempo de Deshidratación

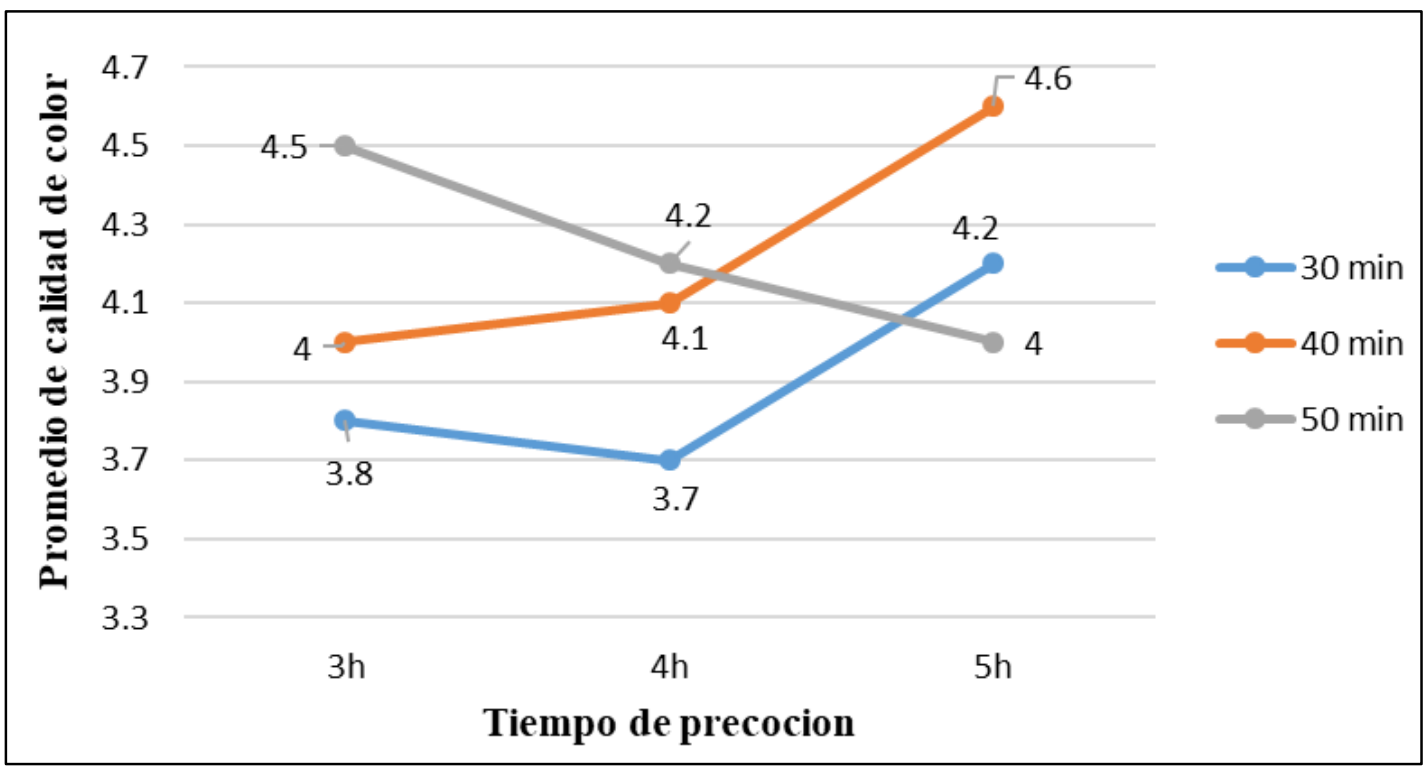

Nota. Elaboración propia.

En la figura 7 se muestran los efectos de interacción del tiempo de precocción vs tiempo de deshidratación para el olor de la menestra precocida y deshidratada, resultado de la prueba de Duncan al $5 \%$. 
Figura 7

Calidad de Olor para efectos de interacción de Tiempo de Pre cocción * Tiempo de Deshidratación

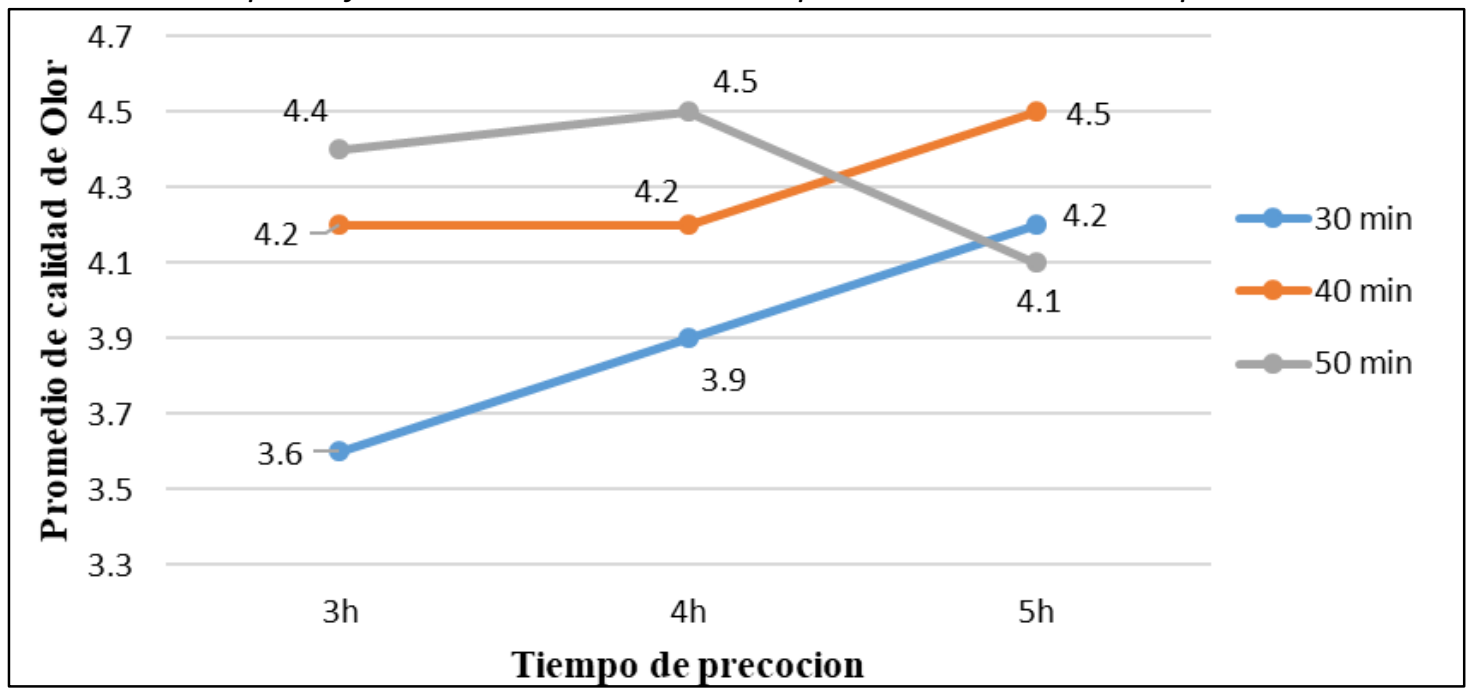

Nota. Elaboración propia.

En la figura 8 se muestran los efectos de interacción del tiempo de precocción vs tiempo de deshidratación para el sabor de la menestra precocida y deshidratada, resultado de la prueba de Duncan al $5 \%$.

\section{Figura 8}

Calidad de Sabor para efectos de interacción de Tiempo de Pre cocción * Tiempo de Deshidratación

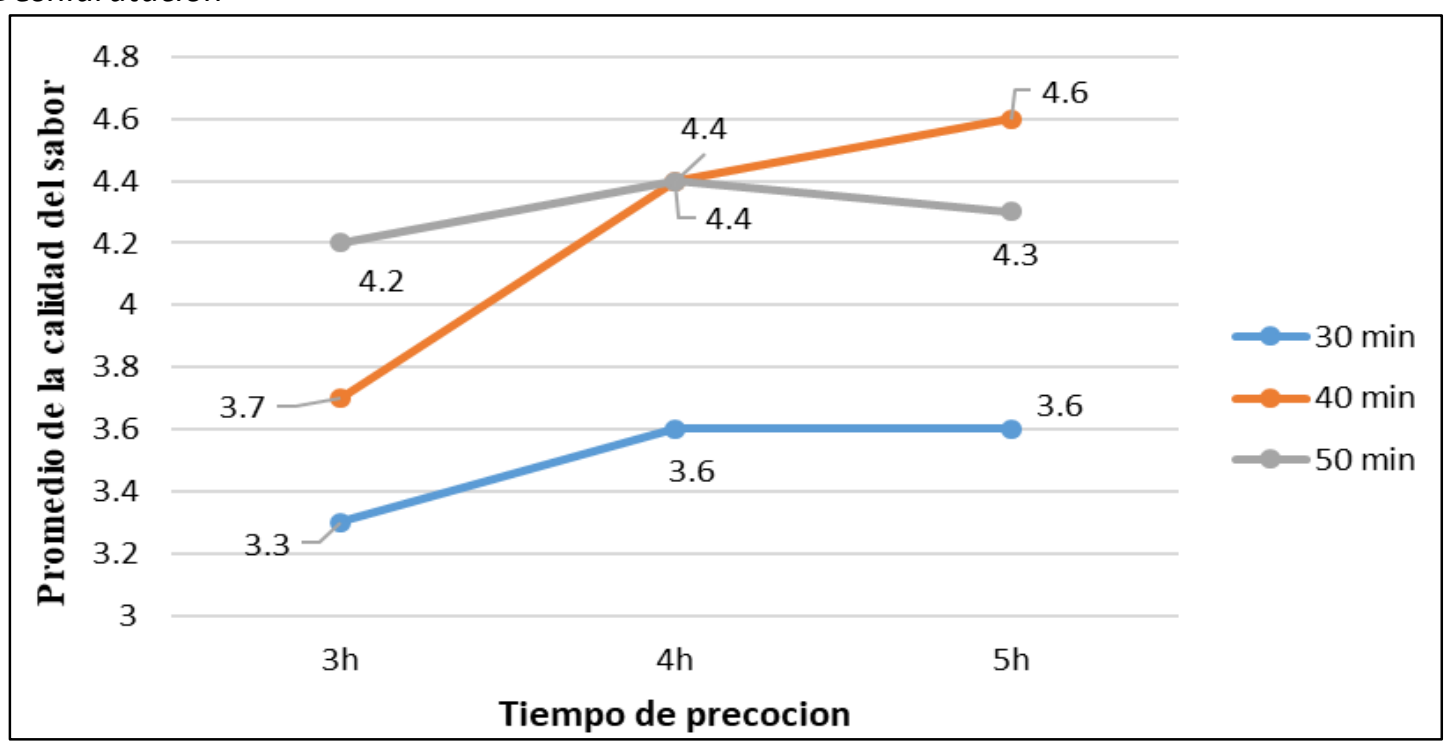

Nota. Elaboración propia.

En la figura 9 se muestran los efectos de interacción del tiempo de precocción vs tiempo de deshidratación para el sabor de la menestra precocida y deshidratada, resultado de la prueba de Duncan al $5 \%$. 


\section{Figura 9}

Duncan al 5\% de Textura para efectos de interacción de Tiempo de Precocción * Tiempo de Deshidratación

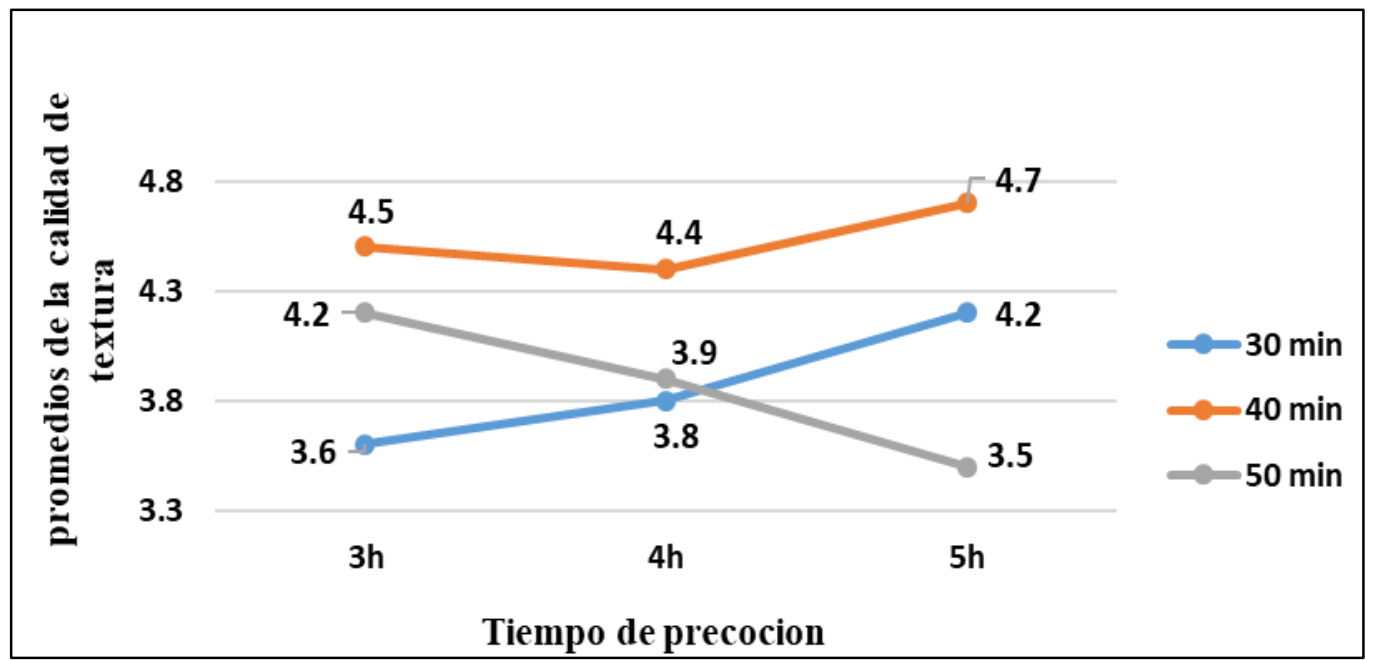

Nota. Elaboración propia.

En la figura 10 se muestran los efectos de interacción del tiempo de precocción vs tiempo de deshidratación para la aceptabilidad de la menestra precocida y deshidratada, resultado de la prueba de Duncan al $5 \%$

\section{Figura 10}

Duncan al 5\% de Aceptabilidad para efectos de interacción de Tiempo de Pre cocción * Tiempo de Deshidratación

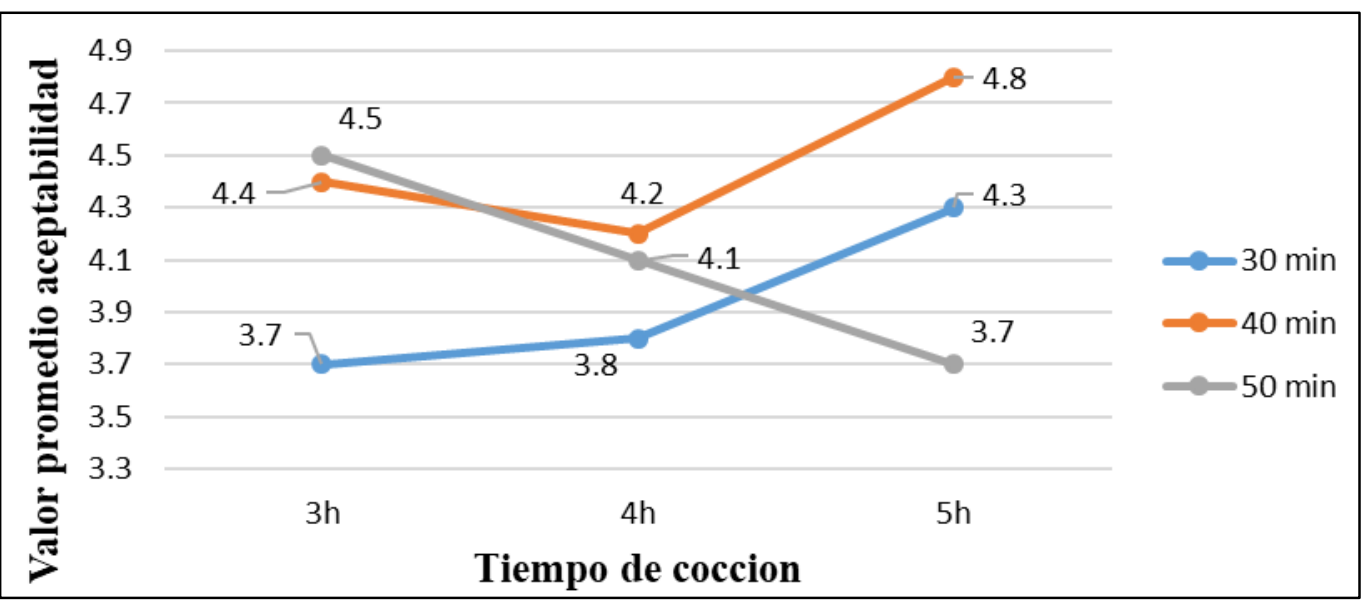

Nota. Elaboración propia.

\section{Discusión}

El remojo cuya variación frente al tiempo se observa en la figura 1, se practicó en el proceso, para ayudar a quitar las pieles, para humedecer y ablandar la semilla y así abreviar el 
tiempo de cocción y reducir el contenido de toxinas. El tiempo necesario para el remojo difiere con las variedades y las especies y también con la duración y condición del almacenamiento (Hurtado, 2005).

El tiempo seleccionado en el proceso de precocción fue de 40 minutos debido a que los granos de frijol castilla muestran la suavidad idónea para ser deshidratado y con características de un producto final de calidad conservando sus propiedades sensoriales, presentando una textura uniforme y lisa; mientras que, a tiempos de 30 y 50 min se obtuvo granos de frijol castilla crudos y excesivamente cocidos, no conservando sus propiedades sensoriales, condición importante para rechazar estos tiempos, considerando que los granos deben presentar una textura uniforme. En cuanto a la deshidratación se determinó que con 40 minutos de pre cocción y un tiempo de 5 horas de deshidratación los granos de frijol castilla obtuvieron un porcentaje de humedad de $12,86 \%$ según la figura 2 , valor que se encuentra dentro de los parámetros establecidos según la NTP 205.015 sobre leguminosas.

Sobre la base a un estudio realizado en un túnel vertical de secado, se estableció que el tiempo de tratamiento en tubérculos almidonosos, como yuca, depende del espesor del material en los anaqueles; la yuca fue secada a $45^{\circ} \mathrm{C}$ en 10 horas. La temperatura considerada como óptima, durante el proceso de secado, para obtener almidón, harina de yuca y tapioca, está entre $50-60^{\circ} \mathrm{C}$ (Molestina, 1957). En la fécula de la Yuca, el proceso de gelatinización comienza a una temperatura de $60^{\circ} \mathrm{C}$ aproximadamente, y termina por debajo de los $80^{\circ} \mathrm{C}$ (Grace, 1977).

La primera etapa del periodo de secado (150 min) está gobernada por el proceso de capilaridad mientras que la segunda ( $270 \mathrm{~min}$ ) está gobernada por el proceso de difusión. Cobrando mayor importancia, por el tiempo de duración, la simulación del proceso mediante la teoría de difusión molecular de agua. La segunda etapa termina cuando el porcentaje humedad se encuentra dentro del parámetro establecido según la NTP 205.015 (2015) para el frijol castilla y esto ocurre a los 300 minutos llegando a tener una humedad final de $0.154 \mathrm{gH} 2 \mathrm{O} / \mathrm{g}$ sólidos seco.

Casp y Abril (1999), indican que el agua es el componente mayoritario de los alimentos en sus dos estados: agua ligada a otros constituyentes y agua libre, móvil, de volumen y estructura variables y fáciles de extraer (Tosello, 1955). La elevación de la temperatura acelera la evaporación superficial de esta agua hasta que se produce una verdadera vaporización a $100{ }^{\circ} \mathrm{C}$, así como favorece la conversión en agua libre de agua parcialmente ligada. Los vegetales frescos tienen una corta vida útil, y están expuestos a condiciones que destruyen su calidad en un corto periodo de tiempo, antes de ser cocidos y consumidos. Por este motivo y por la dependencia estacional del cultivo y recolección de los vegetales se hace necesario la aplicación de tecnologías de la preservación, combinando el mantenimiento de sus características (nutricionales y sensoriales) y alargando su vida útil (Geankoplis, 1998).

La curva representada en la figura 3 refleja los periodos de secado del frijol castilla en el proceso de secado.

El tiempo que se determinó para el proceso de precocción es de 40 minutos debido a que los granos de frijol castilla en ese tiempo muestran la suavidad adecuada para ser deshidratado y obtener un producto final de calidad conservando sus características sensoriales, presentando 
una textura uniforme y lisa. Mientras que, a tiempos de 30, y 50 se obtuvieron granos de frijol castilla crudos y excesivamente bien cocidos respectivamente, no conservando mejor sus propiedades sensoriales, condición importante para rechazar estos tiempos, pues los granos deben presentar una textura uniforme. Por $2.500 \mathrm{~kg}$ de frijol precocido se obtiene $0,974 \mathrm{~kg}$ de frijol deshidratado y se necesitan 5 horas para obtener la deshidratación de esa cantidad de frijol castilla (Tabla 1).

El porcentaje de humedad presenta diferencias significativas (sig. <0,05), al utilizar diferentes tiempos de precocción y diferentes tiempos de deshidratación; también se observan diferencias cuando se combinan los tiempos de precocción con los tiempos de deshidratación (Tabla 3); el tiempo de rehidratación presenta diferencias significativas (sig. $<0,05$ ), al utilizar diferentes tiempos de precocción y diferentes tiempos de deshidratación o la interacción de ambos (Tabla 8). Las menores valoraciones de tiempo de rehidratación se logran con un tiempo de precocción de $40 \mathrm{~min}$ y $50 \mathrm{~min}$ con diferentes tiempos de deshidratación; sin embargo, a un tiempo de precocción de 40 min y a un tiempo de deshidratación a $5 \mathrm{~h}$ el frijol castilla mantiene mejor la mayoría de sus características organolépticas (Figura 5). Diversos investigadores han reconocido que el grado de rehidratación es dependiente de la composición original del producto, condiciones del proceso y preparación, así como de la extensión del desorden químico y estructural provocado por el secado (Okos et al, 1992).

El porcentaje de proteínas que presenta la materia prima frijol castilla es de $19,70 \%$ y la menestra precocidad y deshidratada a partir de frijol castilla es de $22,14 \%$, esta cantidad tiene una mínima variación de merma alrededor de 0,36 con respecto a la indicada por la T.P.C.A las cuales indican $22.5 \%$ de proteínas en este producto (Tabla 12). Nadal (2004), indica que la calidad del grano depende de la variedad y el tipo de suelo.

La mayor valoración en la aceptabilidad de la menestra precocida y deshidratada se logra a un tiempo de precocción de 40 min y $5 \mathrm{~h}$ de tiempo de deshidratación, siendo estadísticamente diferente a las demás combinaciones (figura 10). Los vegetales y hortalizas representan un parte importante de la dieta equilibrada, gracias a sus aportes de vitaminas, minerales, bajos en grasa y proporcionando a su vez color, sabor y textura. Varios estudios han demostrado que reducen el riesgo a padecer algunos tipos de cáncer, gracias a sus altos contenidos en fibra y antioxidantes (Kala y Prakash et al, 2006).

\section{Conclusiones}

El tiempo se determinó que la rehidratación del producto final de la menestra precocida y deshidratada fue de 7 minutos, también la humedad óptima de la menestra precocida y deshidratada después del proceso de secado fue de 12,86 \%, valor que se encuentra dentro del parámetro establecido en requisitos de la Norma técnica: NTP 205.015:2015 para Leguminosas como el Frijol.

Las características fisicoquímicas de la menestra precocida y deshidratada a partir de frijol castilla reportó los siguientes valores: energía total 356,57 Kcal/100; humedad 12,86\%; cenizas 2,51 \%; proteínas 22,14\%; grasa total 3,61\%; carbohidratos totales $58,88 \%$; fibra $5,8 \%$; $\mathrm{pH}$ a $28^{\circ} \mathrm{C}$ 6,6 y acidez $0,18 \%$ expresada como ácido sulfúrico. Valores que se encuentran con una pequeña variación según la T.P.C.A. 
La carga microbiana resultante de los análisis microbiológicos a la muestra final de menestra precocida y deshidratada fue en Aerobios mesofilos $12 \times 10 \mathrm{ufc} / \mathrm{g}$; Mohos $12 \mathrm{ufc} / \mathrm{g}$; Levaduras 0 ufc/g; Coliformes totales <3 NMP/g; salmonella sp (Ausencia/25g); la población microbiana se mantuvo dentro de los límites establecida por la NTP № 071 - MINSA/DIGESA-V.01.

Las características sensoriales de la menestra precocida y deshidratada a partir de frijol castilla que lleven a un producto aceptado por el consumidor fue de 40 minutos de precocción y 5 horas de deshidratado en cuanto a los mejores atributos en color, sabor, textura y aceptabilidad.

\section{Referencias bibliográficas}

Casp, A. y Abril, J. (1999). Procesos de conservación de alimentos. Ed. Mundiprensa y A. Madrid Vicente. Madrid, España.

Chacon k, (2010); Evaluación de rendimiento de grano y otras características agronómicas de cinco líneas promisorias de frijol caupí (Vigna unguiculata (I.) walp), en el sector de Popán AltoDistrito de Zaña -Lambayeque. TESIS para optar el título de Ingeniero agrónomo, Universidad Nacional Pedro Ruiz Gallo Lambayeque - Perú.

Fang Y, Selomulya C y Chen XD, (2007). On measurement of food powder reconstitution properties. Drying Technology, 26(1): 3-14.

Geankoplis CJ, (1998). Procesos de Transporte y Operaciones Unitarias. 3a ed. CECSA. México DF.

Grace, M. (1977). "Elaboración de la Yuca". Organización de las Naciones Unidas para la Agricultura y la Alimentación. Roma. 162 p. (Colección FAO: Producción y Protección Vegetal № 3).

Hurtado M; Márquez A; (2005). Desarrollo y evaluación de croquetas prelistas congeladas utilizando granos de fríjol (Phaseolus vulgaris). Trabajo de grado para optar al Título de INGENIERA DE ALIMENTOS de la Universidad de la Salle. Facultad de Ingeniería de Alimentos. Bogotá.

Kala, Arunachalam Y Prakash, Jamuna (2006). The comparative evaluation of the nutrient composition and sensory attributes of tour vegetables cooked by different methods. International Journal of Food Science and Technology, 41, 163-171.

Molestina O, (1957). La yuca. Revista del Consorcio de Centros Agrícolas (Ecuador). 16(87): 5-8.

Nadal, S; Moreno M. T; Cubero, J. I. (2004). Las leguminosas, grano en la agricultura moderna. Ediciones Mundiprensa. España

Norma Técnica Peruana (NTP) (2015). Leguminosas. Frijol. Requisitos NTP 205.015.

Norma Técnica Sanitaria (NTS) (2008). Norma que establece los criterios microbiológicos de calidad sanitaria e inocuidad para los alimentos y bebidas de consumo humano. NTS № 071 MINSA/DIGESA. V.01.

Okos, M., Narsimhan, G., Singh, R.P., Weitnauer, A. (1992). Deshidratación de alimentos. Manual de Ingeniería de Alimentos, eds. Nueva York, p.437-562.

Tosello A, y Veiga A. de A. (1955). Ensaios sôbre desidratacao de vegetais. Bragantia, 14(21): $215-$ 223

Vargas R, Villamil, O (2012); Caracterización fisicoquímica y nutricional de la harina de frijol caupí (Vigna unguiculata l.) cultivado en el departamento del Tolima. Trabajo de grado para optar el título de ingeniero Agroindustrial Universidad del Tolima Facultad de Ingeniería Agronómica programa de Ingeniería agroindustrial IBAGUE. 